\title{
Thermal sensations and comfort investigations in transient conditions in tropical office
}

\begin{abstract}
The study was done to identify affective and sensory responses observed as a result of hysteresis effects in transient thermal conditions consisting of warm-neutral and neutral warm performed in a quasi-experiment setting. Air-conditioned building interiors in hothumid areas have resulted in thermal discomfort and health risks for people moving into and out of buildings. Reports have shown that the instantaneous change in air temperature can cause abrupt thermoregulation responses. Thermal sensation vote (TSV) and thermal comfort vote (TCV) assessments as a consequence of moving through spaces with distinct thermal conditions were conducted in an existing single-story office in a hot-humid microclimate, maintained at an air temperature $24{ }^{\circ} \mathrm{C}( \pm 0.5)$, relative humidity $51 \%( \pm 7)$, air velocity 0.5 $\mathrm{m} / \mathrm{s}( \pm 0.5)$, and mean radiant temperature (MRT) $26.6{ }^{\circ} \mathrm{C}( \pm 1.2)$. The measured office is connected to a veranda that showed the following semi-outdoor temperatures: air temperature $35{ }^{\circ} \mathrm{C}( \pm 2.1)$, relative humidity $43 \%( \pm 7)$, air velocity $0.4 \mathrm{~m} / \mathrm{s}( \pm 0.4)$, and MRT $36.4{ }^{\circ} \mathrm{C}( \pm$ 2.9). Subjective assessments from 36 college-aged participants consisting of thermal sensations, preferences and comfort votes were correlated against a steady state predicted mean vote (PMV) model. Local skin temperatures on the forehead and dorsal left hand were included to observe physiological responses due to thermal transition. TSV for veranda-office transition showed that no significant means difference with TSV office-veranda transition were found. However, TCV collected from warm-neutral $(-0.24, \pm 1.2)$ and neutral-warm ($0.72, \pm 1.3)$ conditions revealed statistically significant mean differences $(\mathrm{p}<0.05)$. Sensory and affective responses as a consequence of thermal transition after travel from warmneutral-warm conditions did not replicate the hysteresis effects of brief, slightly cool, thermal sensations found in previous laboratory experiments. These findings also indicate that PMV is an acceptable alternative to predict thermal sensation immediately after a down-step thermal transition ( $\leq 1 \mathrm{~min}$ exposure duration) for people living in a hot-humid climate country.
\end{abstract}

Keyword: Semi-outdoor and indoor; Tropical microclimate; Thermal transition; Thermal perception; Skin temperatures; Thermal preference; Thermal sensation vote; PMV; Hysteresis effect 